\title{
Changes in Muscles and Tendons Due to Neural Motor Disorders: Implications for Therapeutic Intervention
}

\author{
A.L. Hof ${ }^{\dagger}$ \\ Department of Medical Physiology, University of Groningen and Laboratory of Human \\ Movement Analysis, University Hospital Groningen, The Netherlands
}

\begin{abstract}
Patients with an upper motor neurone syndrome (CP) suffer from many disabling primary symptoms: spasms, weakness, and loss of dexterity. These primary 'neurogenic' symptoms often lead to secondary disabilities, muscle contractures, and tertiary effects, bone deformations. A common symptom of $\mathrm{CP}$ is hypertonia, with the consequence that the involved muscles remain in an excessively shortened length for most of the time. As a normal reaction of the muscle tissue, the number of sarcomeres is reduced and the muscle fibers shorten permanently: a contracture develops. A possible second type of contracture is that normal muscle lengthening along with bone growth is affected. Current treatments for the secondary effects include (1) reduction of muscle force, (2) lengthening of the muscle fibers by serial plaster casts, and (3) surgical lengthening of tendons or aponeurosis. The choice of treatment depends on the cause of the functional deficit. Bone tissue also adapts itself to abnormal forces, especially in the growth period. The hypertonias or contractures of $\mathrm{CP}$ so may give
\end{abstract}

\footnotetext{
${ }^{\dagger}$ Address for correspondence:

Institute of Human Movement Sciences

University of Groningen, PO Box 196

9700 AD Groningen, The Netherlands

Tel: (31) 50363 2645; fax: (31) 503632751

e-mail: a.l.hof@med.rug.nl
}

rise to bone malformations that interfere with function (e.g. femur endorotation) or may reduce the action of muscles by changing the lever arm (e.g. ankle varus). Although prevention should always be preferred, a timely surgical intervention cannot always be avoided. The differences in treatment for the various groups require and justify an extensive laboratory investigation, including EMG recordings in gait, measurement of passive elastic properties, and long-term observation of the hypertonia.

\section{KEYWORDS}

hypertonia, casting, equinus, contracture, cerebral palsy, tendon elasticity

\section{INTRODUCTION}

Adaptation and plasticity in the nervous system is the main subject of this issue, but muscles, tendons, and bones show these effects as well. In contrast to the nervous system, however, the adaptation and plasticity in muscle and bone generally are detrimental and should be avoided, minimized, or corrected as much as possible. The case of cerebral palsy (CP) provides a good illustration. The primary cause is purely neurologic: brain damage from perinatal age. All other effects, muscle and tendon shortenings and bone deformations, are secondary. That does not imply that 
they are less important. In many cases, the secondary effects are more crippling than the original disease itself. As an illustration, the problem of equinus gait will be considered. To show the problems in $\mathrm{CP}$, first the normal activity of the ankle plantarflexors in walking will be discussed.

\section{Normal walking}

The ankle plantarflexors comprise three muscles of which the monoarticular soleus muscle is the strongest. The gastrocnemius muscle, divided into a medial and a lateral head, is biarticular. It not only plantarflexes the ankle but also flexes the knee. In walking, all three muscles are strongly active in the stance phase (Fig. 1). During stance, the force increases gradually from early stance during foot dorsiflexion (second rocker) to reach a maximum at the time of contralateral heel contact. It then declines very rapidly to zero at toe-off.

Maximum force is very considerable and amounts to about three times body weight in normal subjects. This is about $50 \%$ of the peak force that can be generated by these muscles in top speed running. In contrast, the plantarflexor activity in the swing phase of walking is virtually zero. There is a moderate dorsiflexor (tibialis anterior) activity to keep the ankle flexed during swing.

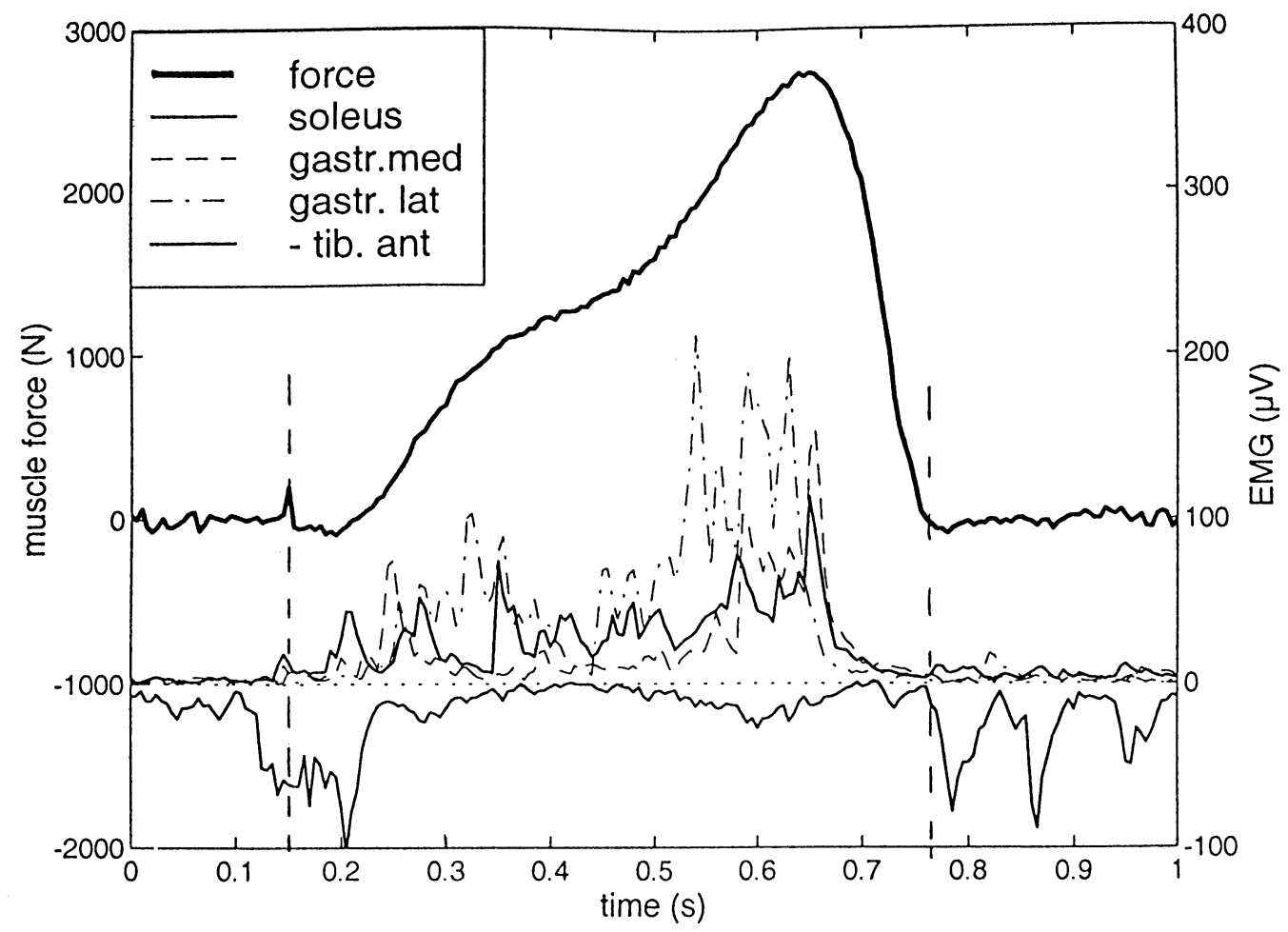

Fig. 1: Force and EMGs of the human plantarflexor muscles during walking at normal speed. Stance period is between the vertical dashed lines. During stance, the major plantarflexors, soleus and both heads of gastrocnemius, all are active. Dorsiflexor tibialis anterior (EMG drawn downward) is active in swing to keep the foot from the ground. There is a short period of cocontraction at the transition from swing to stance. 


\section{Primary effects of CP}

The primary effects of CP can be separated into excess symptoms and deficit symptoms (Young \& Wiegner, 1987; Young, 1994; Becher, 2000). The deficit symptoms are paresis, loss of dexterity, and reduced ability to perform isolated movements. For our discussion, the excess symptoms spasticity, cocontraction, and hypertonia are the most important. Both the spontaneous spasms and the cocontraction can cause hypertonia: an increased level of muscle activity over very long times that cannot be sufficiently counteracted by the antagonists.

In $\mathrm{CP}$, biarticular muscles are usually more affected than mono-articular muscles by hypertonia and spasticity. In the example of the ankle plantarflexors, therefore, the gastrocnemius may be excessively active, even when it should be at rest, whereas the tibialis anterior muscle (which is much weaker anyhow) has reduced force. The foot will then stay continually in the plantarflexed position, with shortened triceps surae. This condition is known as the equinus foot. During the stance phase of walking, this has the effect that the patient continually walks on his toes. In swing, the ongoing ankle plantarflexion makes the effective leg longer, which has to be compensated in some way to avoid stumbling. The primary pathology of $\mathrm{CP}$ can thus already lead to an equinus foot.

\section{SECONDARY EFFECTS OF CP}

\section{Muscle fibers}

Muscle fibers adapt quickly to changing circumstances. There are three mechanisms.

1. The first mechanism is adaptation to force.

This is also known as the strength training effect. When a muscle is activated maximally for a short time a day, it will respond by increasing strength. The fibers become thicker, the number of parallel contractile filaments increase, and muscle force increases. In contrast, when a muscle is not used, as in paresis, it is wasted very soon.

2. The second mechanism is adaptation in speed. Muscle fibers can either be slow (type I) or fast (type I). Many physiologists have studied the development of these properties. These properties are in part genetically determined, but they express themselves only when the correct activation patterns occur in daily life. Motor units 'born to be fast' require a 'fast' stimulus pattern to become fast: a high firing frequency in short periods of activity. The abnormal activation of the muscles in $\mathrm{CP}$ has a direct effect on the muscle fiber composition. Activity is almost continuous and firing frequencies are never high. The consequence is that muscles become slower (Rose \& McGill, 1998). Together with the spasticity, which implies a reduced ability to suppress muscle activation and with a possible weakening due to atrophy, this slowness can result in a considerably reduced 'modulation' of muscle force development.

3. The third mechanism in muscle is adaptation in length. In the growing child, the bones increase in length. The normal reaction of muscle tissue is to increase in length as well. This means that the number of sarcomeres in series increases in a muscle fiber and it adapts itself to the range of lengths it is subjected to. This is a normal and physiological process.

However, when a muscle due to hypertonia is continuously kept in a shortened position, the muscle fibers may adapt to this decreased length and become unable to lengthen to normal lengths. This condition is termed a contracture. The course of events is thus (Fig. 2) that abnormal motor control leads to hypertonia, and a hypertonia which cannot be counteracted by the antagonists 


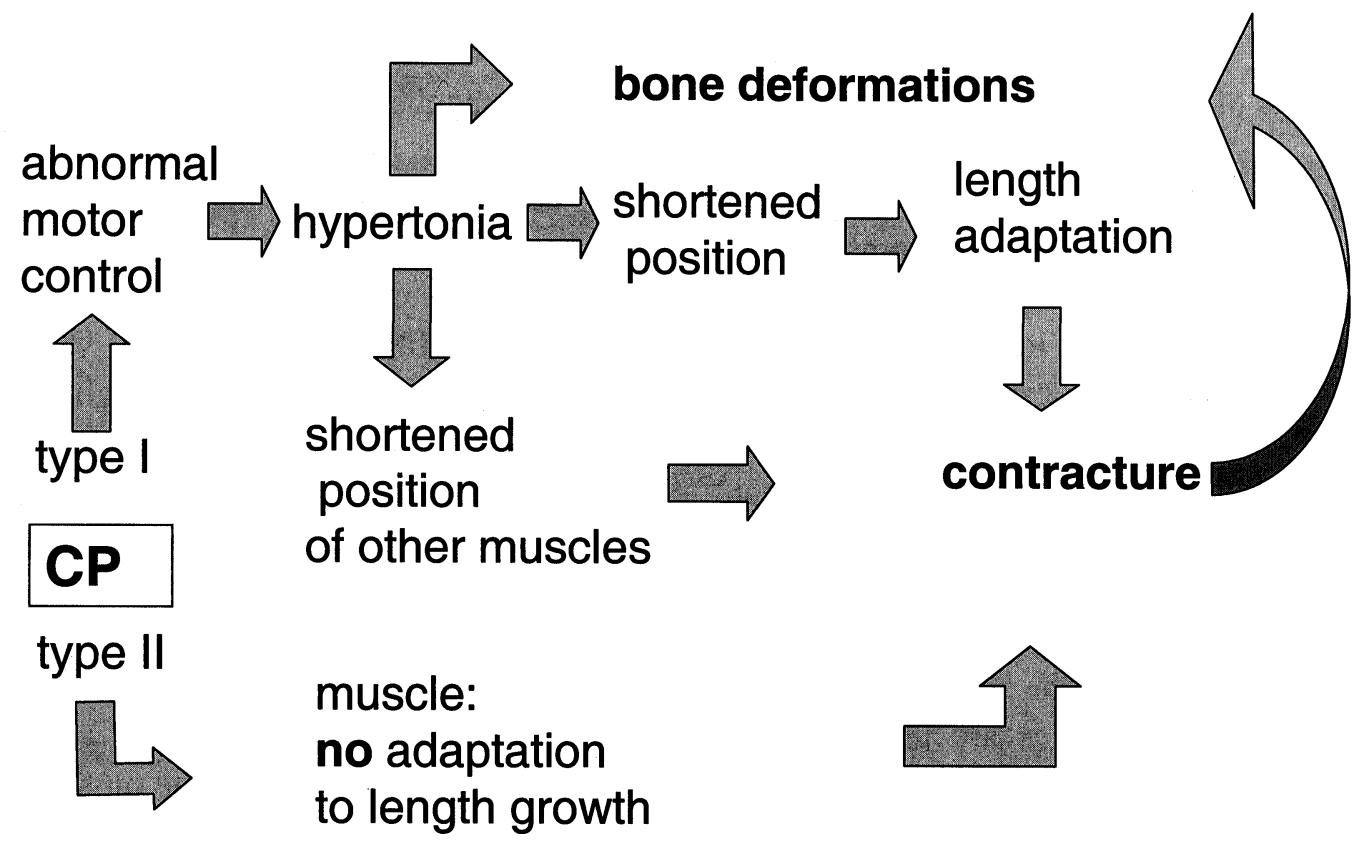

Fig. 2: Interaction of processes leading from cerebral palsy $(\mathrm{CP})$ to contracture and body deformations.

leads to a state of continuous shortening of the muscle. This active shortening can then result in a permanent length adaptation, a contracture. When, for example, the gastrocnemius generates so much force that the antagonist tibialis anterior cannot adequately counteract the ankle will remain continuously in an equinus position. This position can become fixed into a contracture.

The chain of events can also run indirectly. The same gastrocnemius hypertonia with a resulting equinus can lead to a contracture of the soleus, which is passively shortened, without being spastic or hypertonic. Still another possibility is that the contracture is the result of compensatory actions. A gastrocnemius hypertonia may lead to equinus and knee bending. To compensate for this, a posture with flexed knees and hips (crouched gait) is assumed, and a contracture of the hip flexors may result.

\section{Contracture}

It is appropriate to discuss the mechanical correlates of contracture here. A muscle-tendon unit can mechanically be considered as a threecomponent system (Fig. 3). The contractile component, which can grossly be identified with the muscle fibers, can develop force over a certain range of lengths (Fig. 4, dashed line). The maximum force depends on the level of activation. Parallel to this element is a passive elasticity, representing the connective tissue around and next to the muscle fiber (Fig. 4, continuous line). Over most of the range of lengths its force is low, but beyond a certain limit, passive force increases very steeply. This increase of force serves to protect the contractile muscle fibers from overstretching.

In gastrocnemius and soleus, the muscle fibers are very short: their length $l_{c}$ is some 4 to $6 \mathrm{~cm}$. The remainder of the length, $l_{s}$ of about $30 \mathrm{~cm}$, comprises aponeurosis and tendon. In the scheme in Fig. 3, this is denoted as the series elastic component (SEC). Both the active and the passive force-length relations are displaced therefore to lengths around about $30 \mathrm{~cm}$, see Fig. 5 . The forcelength relations at the left are those of muscle fibers (with length $l_{c}$ ), those at the left for the complete muscle-tendon unit, with length $l=l_{c}+l_{s}$. 


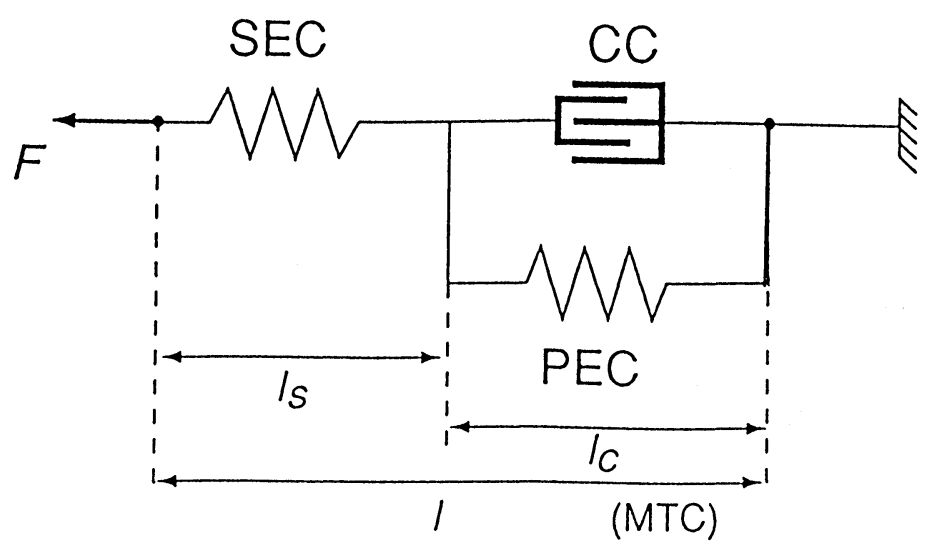

Fig. 3: Schematic diagram of the mechanical components of muscle. The contractile component (CC) with length $l_{c}$ represents the length of the contractile muscle fibers. The muscle fibers and their associated connective tissue also have elastic properties, the parallel elastic component (PEC). In a series with the muscle fibers are the tendon and aponeurosis, represented in the series-elastic component (SEC), with length $l_{s}$. In the human plantarflexors total length $l$ of the muscle -tendon complex is about $30 \mathrm{~cm}$, of which $l_{s}$ is around $26 \mathrm{~cm}$ and $l_{c}$ only $4 \mathrm{~cm}$.

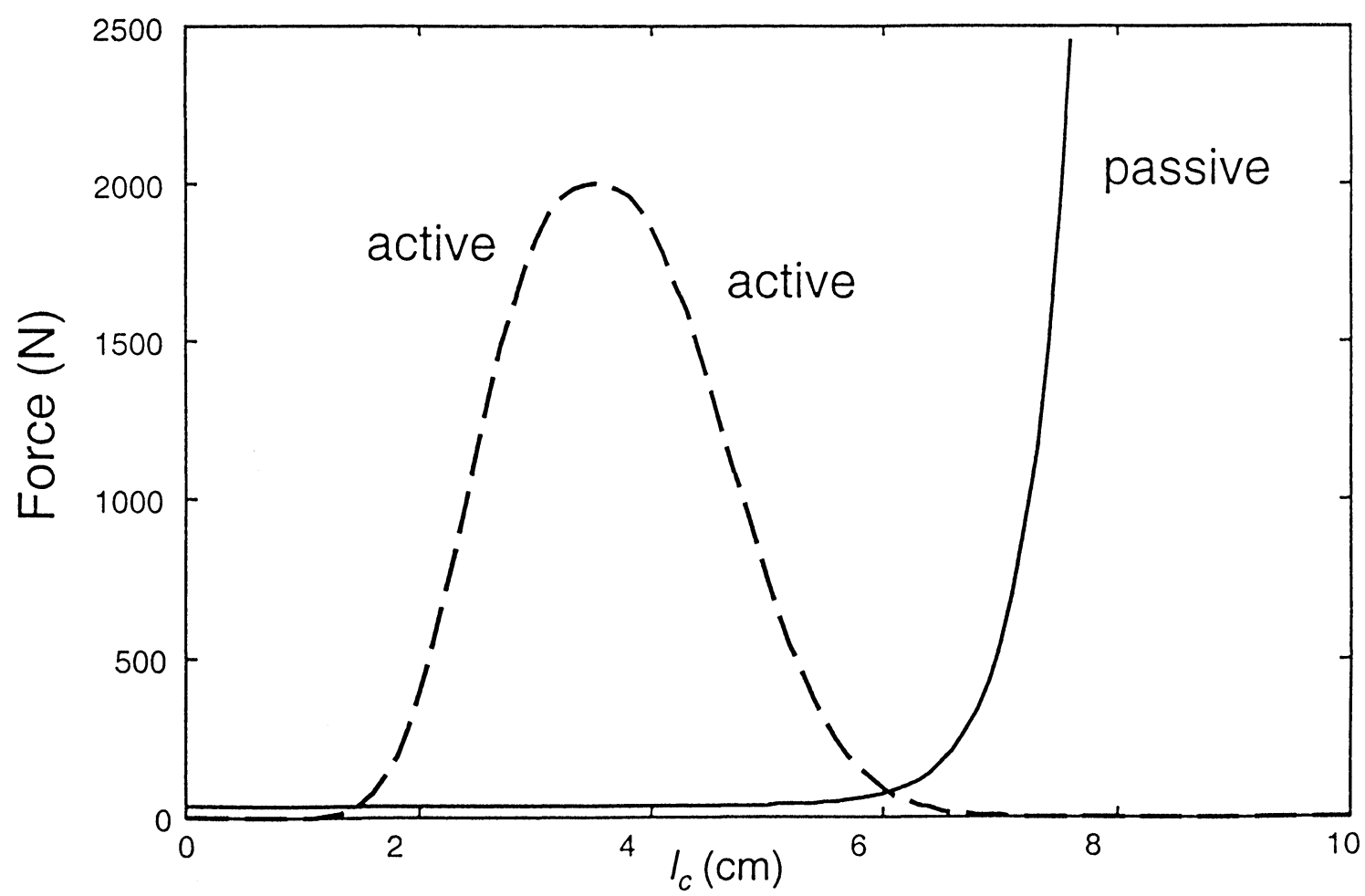

Fig. 4: Force-length relations of $\mathrm{CC}$ (active force, dashed) and PEC (passive force, solid) for the human plantarflexors as a function of $\mathrm{CC}$ length $l_{\mathrm{c}}$. The active force is controlled in amplitude by the muscle activation, peak level can be between 0 and about $5000 \mathrm{~N}$. The curves have been obtained from the same subject as from the walking recording of Fig. 1. 


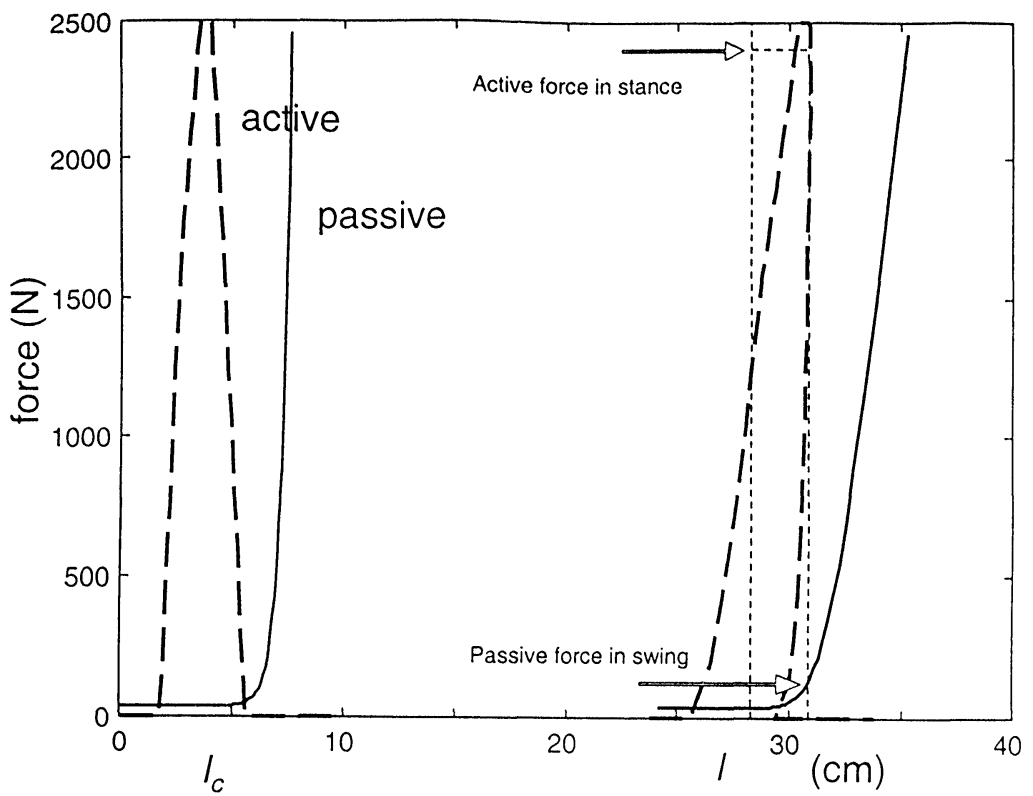

Fig. 5: Force-length relations of CC (active force, - -) and PEC (passive force, - - ) for the human plantarflexors as a function of both CC length $l_{c}$ (at the left) and total muscle+tendon length $l$ (right). Right figure: the range of lengths during walking is indicated by vertical lines (- -). The muscle+tendon figures at the right are somewhat inclined to the right, due to the compliance of the series elastic component. Upper arrow: maximum required active force during stance. Lower arrow: remaining passive force during swing. This force, together with the weight of the foot, is countereacted by the dorsiflexor muscles. In normal muscle, the available active force is amply sufficient over the whole length range, while the passive force is small.

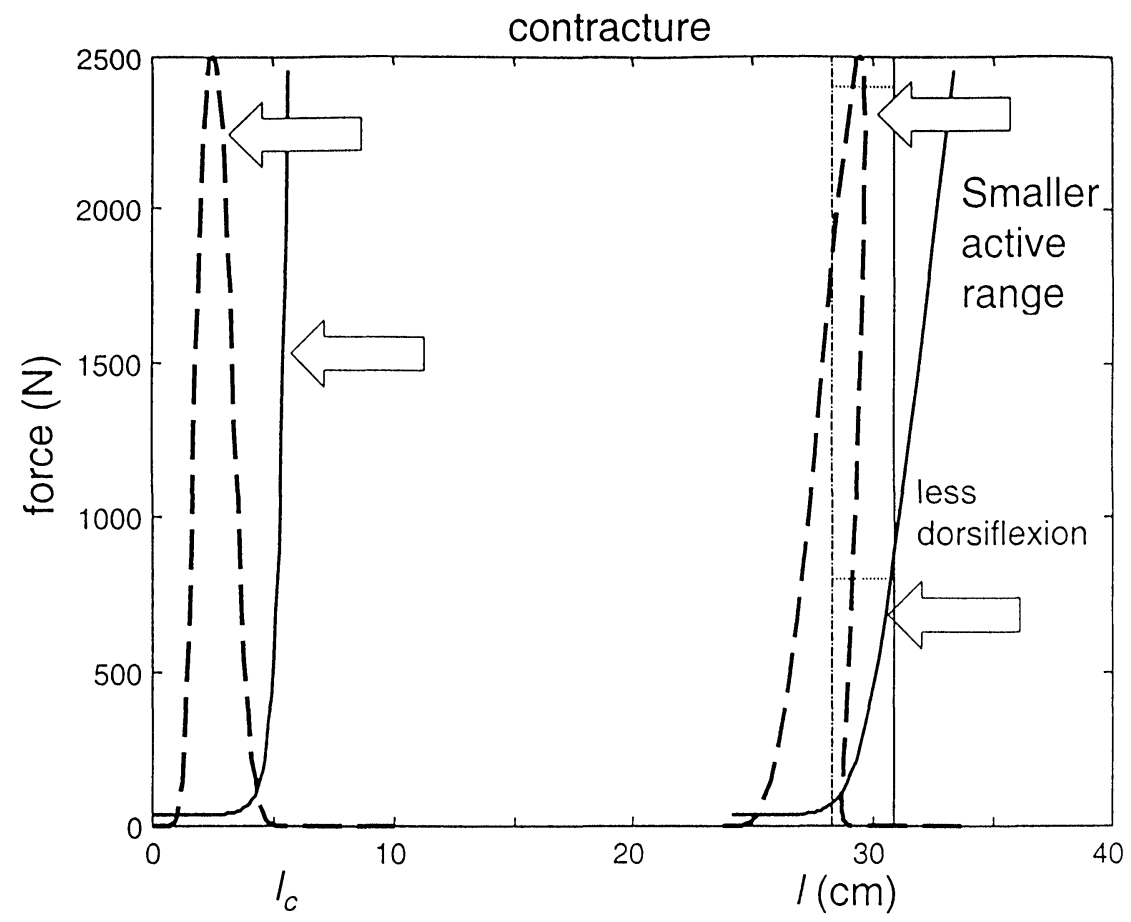

Fig. 6: As Fig. 5, but now for a subject with a contracture. Both the active force and passive force-length relations are shifted to shorter lengths. This has two consequences. First, the required active force is now available only at the shorter muscle lengths. Second, the passive force has so much increased that passive dorsiflexion is reduced. Both lead to an equinus (tiptoe) gait. 
It should be noted that the tendon is somewhat compliant: it is stretched at higher forces. This can be seen from the less steep slope of the curves at the right. Length changes due to the normal range of movements in walking are also given in this figure. In normal subjects, the passive force is low over the usual range of movement and the active force is high. The maximum force of three times body weight during walking can be easily achieved in normal subjects. The low passive force of the plantarflexors will be counteracted by active force from the tibialis anterior. This muscle can generate a force of up to about $800 \mathrm{~N}$, which is amply sufficient to dorsiflex the foot over the whole range.

When a contracture has developed, muscle fibers have become too short because of a diminished number of sarcomeres. This is reflected in the active force-length relation, which has become narrower and has shifted to the left (Fig. 6). As long as the tendon length does not change, the same can be seen at the joint. This results in a decrease of the active range: the muscle cannot generate high forces at dorsiflexed positions.

Together with the muscle fibers, connective tissue also decreases in length. At the ankle, the passive range of motion is diminished, and that is what is usually measured in order to define a contracture (Tardieu et al., 1982a,b). All these effects have been studied and reproduced in experimental animals (Tabary et al., 1972).

Several problems still remain, however. Why do the muscle fibers adapt to decreased length and not the tendon? The answer seems to be a matter of time: muscle tissue adapts itself in the course of weeks, but tendon does so in the course of months. But eventually, the tendon should also increase in length as a result of bone growth. Another question is whether passive elasticity, a property of connective tissue, always follows changes in muscle fiber length. This is usually assumed, but the reason why is unclear.

With the mechanical model as presented, it is simple to assess the effect of a surgical procedure, such as lengthening of the Achilles tendon (Fig. 7) When the tendon is increased in length, the curves move to the left. The good result is that the passive force is reduced and the passive range improves. The bad result can be that the active force is strongly reduced, and that only part of the active force-length relation is used now. These effects become worse with increased lengthening. The effect can be cumulative: with too much lengthening the force becomes very low; the muscle is no longer is use and the tendon, which should grow and adapt to the muscle fiber, remains too thin and too compliant. A more extensive discussion of fasciotomy and the interaction of muscle fiber with connective tissue in general can be found in Huijing (1999).

\section{Group II contractures}

The contracture in the form as discussed above is a consequence of a normal process of muscle length adaptation in response to abnormal muscle activation. This type is known as a Group I contracture (Tardieu \& Tardieu, 1987).' Another type of conracture, Group II, has a different origin. As a consequence of the $\mathrm{CP}$, the normal process of length adaptation can be deranged, meaning that the muscle no longer 'grows with the bone'. The exact mechanism of this neurogenic growth disturbance is unknown, but it is a well known clinical phenomenon. In this case, the muscles do not become too short, but the bones become 'too' long. This indicates two quite different pathways leading to contractures.

The continuous tension, due to either the hypertonia itself or to the contracture, finally can lead to deformations of the skeleton. This is especially true in the growing child. The problems can be many: valgus or varus of the foot, malrotations of the tibia or the femur, hip luxations, etc.

${ }^{1}$ Confusingly, Tardieu et al. (1982) used the reversed classification in an earlier paper. 


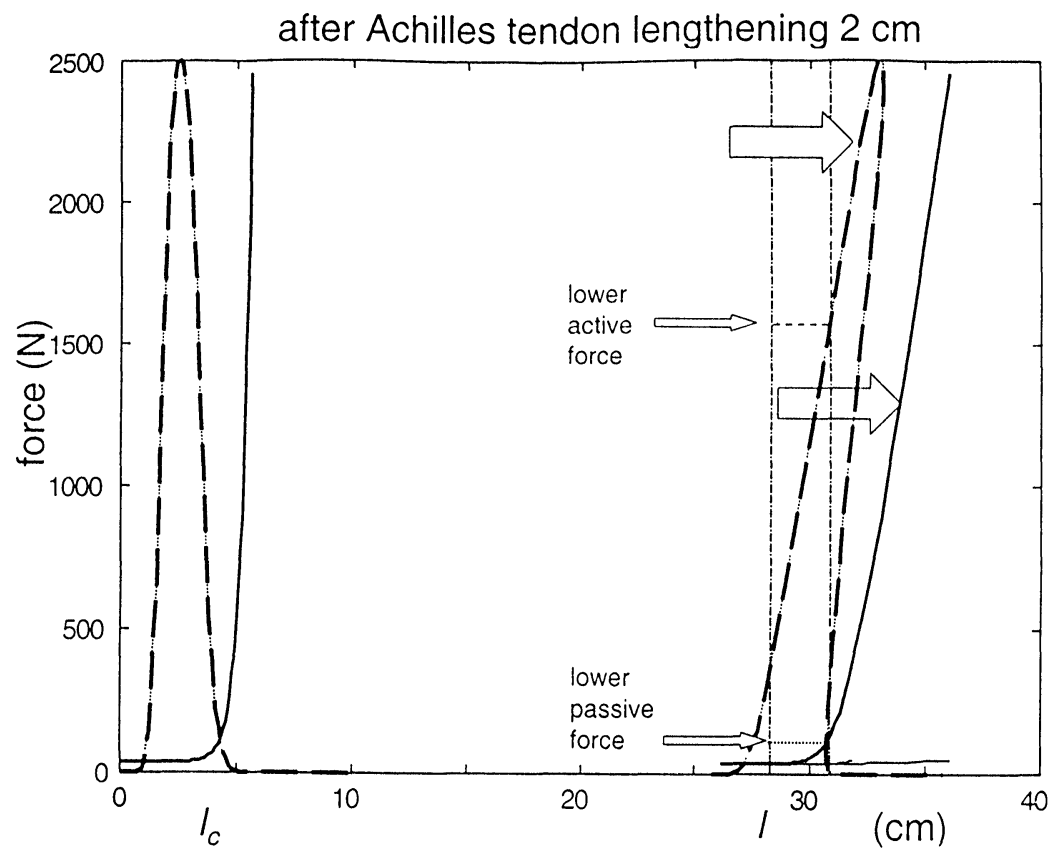

Fig. 7: The contracture has been corrected by $2 \mathrm{~cm}$. Achilles tendon lengthening. The passive force has been much reduced, which is beneficial but also the active force is reduced. When the lengthening has been excessive, this can lead to an inability to walk.

\section{THERAPY}

\section{Reduction of hypertonia}

What can be done against these malformations and maladaptations? Clearly, the best solution would be prevention. For $\mathrm{CP}$, this means to prevent or to reduce the hypertonia and to correct the unequal balance between antagonistic muscles. For this, a number of therapies is available, but each has major side effects.

The first category of treatment tries to reduce spasms and spasticity by acting on the central nervous system. Selective rhizotomy is a major neurosurgical operation, in which the sensory roots of the spinal column are partially transsected. This operation may reduce the spasticity, i.e., the exaggerated reflexes. Baclophen is a GABA antagonist that can be applied intrathecally in the spinal canal, by means of an implanted pump. Both these therapies are quite invasive and should only be used in the most severe cases.

The second category of therapy aims at a reduction of muscle force in selected muscles. A permanent reduction can be attempted by a partial destruction of the nerve endings, either by toxic substances like phenol or alcohol or by heat, thermocoagulation. A problem is that the application must be precisely at the motor endplates as the treatment is not really specific. In principle, the effect is permanent, but reinnervation may occur.

Injection with botulinum toxin has a similar effect, but only temporary. This toxin very selectively inhibits the endplates and is effective during 3 to 6 months. After this period, new endplates on the original muscle have formed. This treatment is becoming increasingly popular because it is selective, the site of injection is less critical, 
and there are few side effects (Russmann et al., 1997; Molenaers et al., 1999; Graham, et al., 2000).

All these treatments aim at a reduction of the muscle force in the selected muscles. The disadvantage is obvious: the active muscle force needed for movement is reduced. This holds even more for the drug Dantrium (Dantrolene sodium). It can be taken orally and gives a generalized reduction of muscle force. It works directly on the actinmyosin crossbridges in muscles. However, it aselectively weakens all muscles, including the paretic muscles and the antagonists of hypertonic muscles.

\section{Redressing contractures}

A second line of defense is trying to prevent or to redress contractures as a consequence of hypertonia. The physiotherapist, in the first place, should be very alert to this problem. Suitable exercises, inducing the child to use the full range of motion in the joint at risk, may postpone more drastic actions. An orthosis can be fitted to restrict joint motion, or a casting can be applied to force the joint so that the muscle is lengthened. When the muscle kept for some time elongated, the number of sarcomeres adapts itself to this longer length. By way of the same adaptation in sarcomere number that causes the problem, it thus can be remedied. The joint angle can be progressively changed in a number of steps (serial casting). Casting during the night can, in some cases, be sufficient.

It will be obvious that all these treatments are effective only in the case of a Group I contracture. When the case is Group II, when the contracture is caused by an inhibited muscle growth, medication to reduce muscle force is useless and casting does not work. (It rather will give strong discomfort.) In those cases, a timely series of surgical interventions is the only possible option.

\section{Surgical interventions}

When contractures have developed and cannto be redressed, and in the case of skeletal deformities, only surgical interventions remain. These can be divided into soft tissue operations, corrections of contractures, and skeletal corrections.

In case of the equinus foot, mere lengthening of the Achilles tendon, which was for many years the favorite option in orthopedic surgery, is not the optimum treatment. Often the gastrocnemius is hypertonic and the soleus normal. A tendon lengthening, therefore, would weaken the active forces of both muscles. If the tendon is lengthened too much, the remedy is worse than the disease. A better option is a partial aponeurotomy of gastrocnemius only (Baumann, 1989). For a biarticular muscle like the rectus femoris, transfer of the distal tendon is also an option as this transforms the muscle from a knee extensor into a knee flexor.

Finally, when skeletal deformations need correction, surgery is the only option. The present opinion is that all corrections are best performed in a single major operation, bones together with the soft tissues, the so-called multilevel surgery (Nene et al., 1993).

\section{DIAGNOSIS}

In order to select the optimal treatment for maladaptation in muscles, tendons, and skeleton, a careful diagnosis is needed. The first question is which muscles are hypertonic. That is, which muscles show an exaggerated or badly timed activity, such that there is a danger of contracture. Next to the clinical examination, which should include a movement observation, an instrumented gait analysis is necessary. In our opinion, an EMG recording, which can show abnormal muscle activity patterns, can be especially helpful. The diagnostic 
problem is not easily solved, however, as it can be quite difficult at times to discriminate between abnormal or spastic activity and compensatory activity. Compensatory activity is in itself abnormal, different from the normal pattern, but it is a sensible action of the individual to correct for other deficiencies. It is certainly a mistake to treat the involved muscles with botulin toxin or to operate their tendons. When the actual reason of the compensation is remedied, the compensation itself is no longer needed and will disappear by itself.

The second question is which muscles already have developed a contracture? It is not easy to discriminate between a muscle that is shortened due to hypertonia-abnormally high activity-or to an already developed contracture-a high passive stiffness. The distinction is quite important for the treatment, however. Hypertonia can be treated with botulin, a contracture might be corrected with a casting. The clinical examination, mainly the assessment of the passive range of motion, is usually not sufficient. An EMG gait recording can be helpful. The best and most direct option is a recording of the moment-angle curve, together with the EMG (Tardieu et al., 1982a, b; Tardieu \& Tardieu, 1987; Becher et al., 1998). In that case, the passive stiffness is determined quantitatively, and whether there is muscle activity can be verified.

When a contracture had already developed, a final discrimination in the diagnosis should be made between a Tardieu Group I and Group II contracture. A difference is that in Group I there is an underlying hypertonia and in Group II there is not. If, according to observations of both the therapist and the parents, there are no spasms or abnormal contractions at day or night, the disorder might be of Group II, contracture due to growth deficit. In such a case, casting is useless, and the only option is a series of operations. In practice, the differentiation is not that easy, and the final proof is often the effect of casting: if a casting has no effect, the patient probably suffers from a Group II contracture.

\section{CONCLUSION}

The conclusions from this brief review can be short and simple. First, prevention is better than cure. Only in part of the cases it will be possible to prevent contractures or skeletal deformations, but every effort should be made to try. In our opinion, this can only be done when $\mathrm{CP}$ children are followed and supported a frequent intervals from a very early age.

Secondly, it should be clear that good diagnostic instruments are important to guide treatment. Observations of movements and a clinical examination of the range of movement are necessary, but they are not sufficient to plan a longtime casting procedure or a multilevel operation with far-reaching consequences.

\section{ACKNOWLEDGEMENT}

I thank Prof. K. Postema, Prof. D. Kernell, and Dr. D. Scrutton for helpful discussions.

\section{REFERENCES}

Baumann JU, Koch HG. 1989. Ventrale aponeurotische Verlängerung des Muskels gastrocnemius. Operative Orthopädie und Traumatologie 1:254-258.

Becher JG, Harlaar J, Lankhorst GJ, Vogelaar TW. 1998. Measurement of impaired muscle function of the gastrocnemius, soleus and tibialis anterior muscles in spastic hemiplegia-A preliminary study. J Rehabil Res Dev 35: 314-326.

Graham HK, Aoki DR, Autti-Rämö I, Boyd RN, et al. 2000. Recommendations for the use of botulinum toxin type A in the management of cerebral palsy. Gait Posture 11: 67-79. 
Huijing PA. 1999. Muscle as a collagen fiber reinforced composite: A review of force transmission in muscle and whole limb. J Biomechanics 32: 329-345.

Molenaers G, Desloovere K, Eyssen M, Decat J, Jonkers I, De Cock P. 1999. Botulinum toxin type A treatment of cerebral palsy: An integrated approach. Eur J Neurol 6 Suppl: S51-S57.

Nene AV, Evans GA, Patrick JH. 1993. Simultaneous multiple operations for spastic diplegia. J Bone Joint Surg 75-B: 488-494.

Rose J, McGill C. 1998. The motor unit in cerebral palsy. Dev Med Child Neurol 40: 270-277.

Russmann BS, Tilton A, Gormley ME. 1997. Cerebral palsy: A rational approach to a treatment protocol, and the role of botulinum toxin on treatment. Muscle Nerve S6: s181-s193.

Tabary JC, Tabary C, Tardieu C, Tardieu G, Goldspink G. 1972. Physiological and structural changes in the cat's soleus muscle due to immobilisation at different lengths by plaster casts. J Physiol 224: 231-234.

Tardieu C, Huet de la Tour E, Bret MD, Tardieu G. 1982a. Muscle hypoextensibility in children with cerebral palsy: I. Clinical and experimental observations. Arch Phys Med Rehab 63: 97-102.

Tardieu G, Tardieu C, Colbeau-Justin P, Lespargot A. $1982 \mathrm{~b}$. Muscle hypoextensibility in children with cerebral palsy: II. Therapeutical implications. Arch Phys Med Rehab 63: 103-107.

Tardieu G, Tardieu C. 1987. Cerebral palsy. Mechanical evaluation and conservative correction of limb joint contractures. Clin Orth Rel Res 219: 63-69.

Young RR. 1994. Spasticity. Neurology 44 Suppl: S12-S20.

Young RR, Wiegner AW. 1987. Spasticity. Clin Orth Rel Res 219: 50-62. 

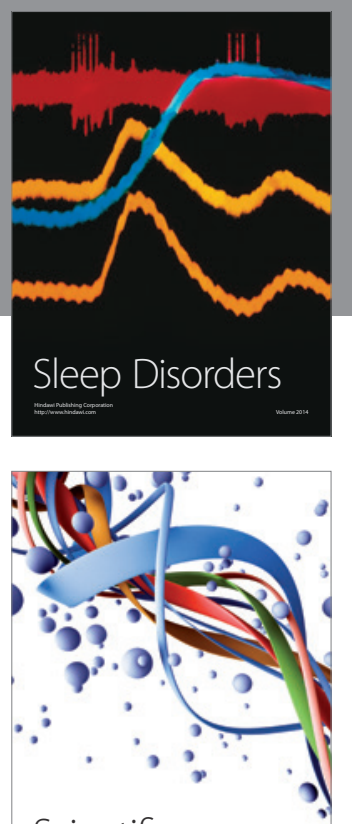

Scientifica
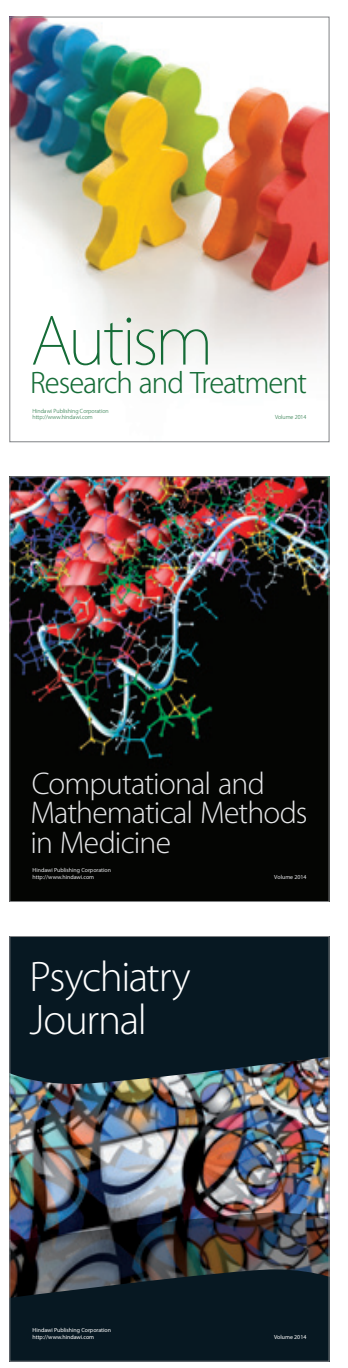
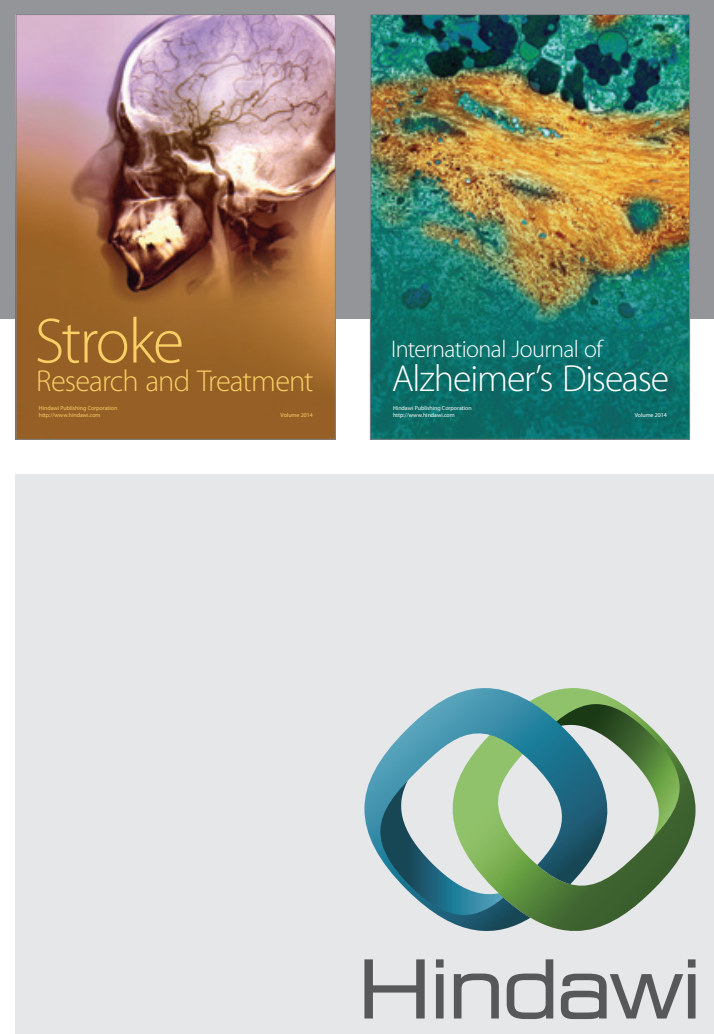

Submit your manuscripts at

http://www.hindawi.com
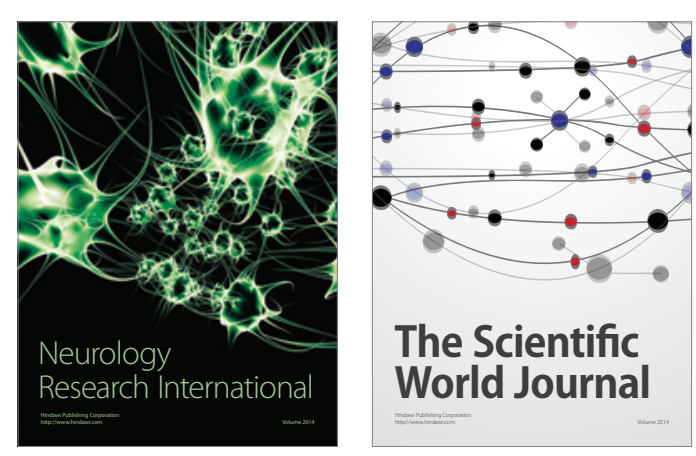

The Scientific World Journal

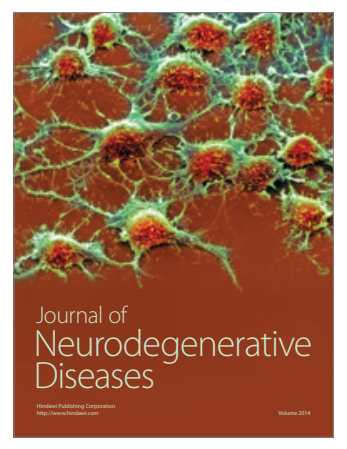

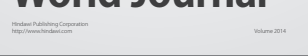

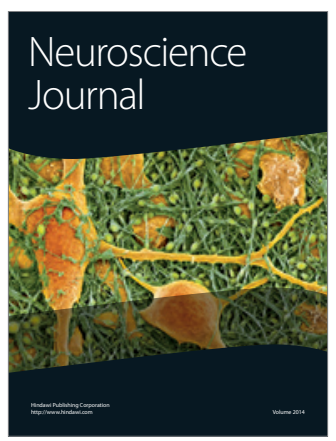

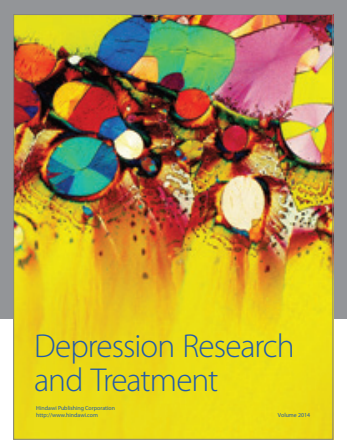
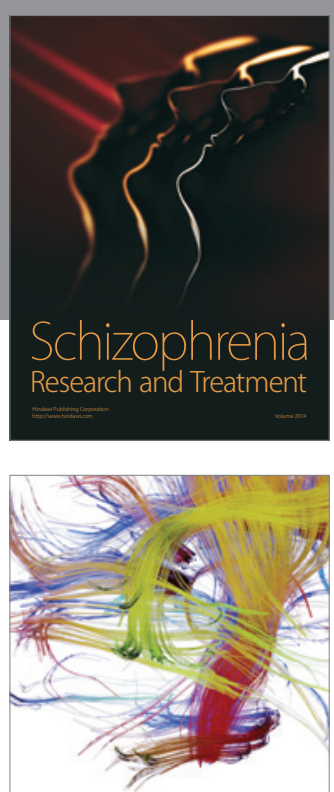

Brain Science

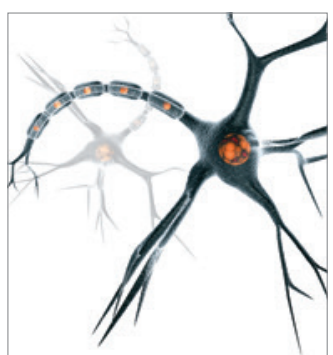

Neural Plasticity
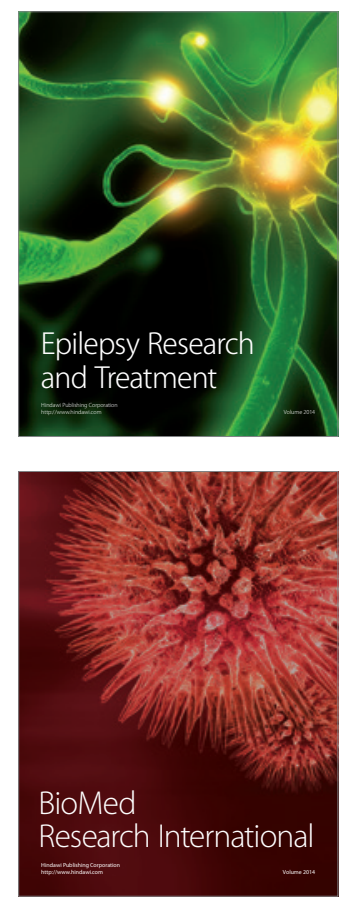

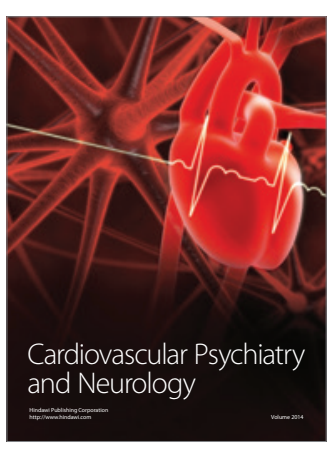

Parkinson's

Disease
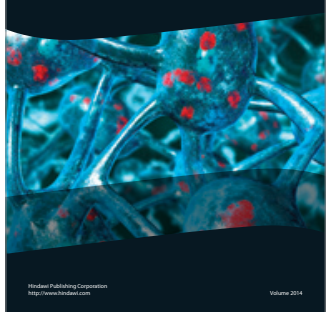